\title{
DATA PROCESSING APPROACHES FOR LUNG CT- IMAGE ANALYSIS UNDER RESOURCE CONSTRAINTS
}

\author{
Aleksandra Vatian, Artyom Lobantsev, Nikita Gorokhov, Mikhail Mirzayanov, Georgii Korneev, \\ Natalia Gusarova and Anatoly Shalyto \\ ITMO University \\ 49 Kronverksky pr., St. Petersburg, 197101, Russia
}

\begin{abstract}
In this paper we consider the methods of the improvement the efficiency of lung tumor classification made by deep convolutional networks in conditions of limited resources. We propose two approaches of the efficiency improvenets. The first is the consideration of the specifics of the brightness distribution of CT images of lung cancer and preprocessing the data according to these specifics. Experiments with the inclusion of preprocessing proposed by this method showed an improvement in the classification efficiency on a small amount of computing resources. The second is the use of an improved network training method for the task of domain adaptation, which includes the integration of a specific loss function into the network architecture. That loss function aligns the latent feature space of the network trained on source large dataset of CT scans with the latent feature space of the target small dataset, where the network will be used for inference. The integration of this method has shown comparable results with the traditional fine-tuning method in a noticeably smaller amount of time and on the same hardware resources.
\end{abstract}

\section{KEYWORDS}

Lung Tumor, Resource Constraints, Domain Adaptation, Data Preprocessing, CT-Images

\section{INTRODUCTION}

In recent years, medical images have become one of the leading sources of diagnostic and therapeutic information, especially for such severe and fast diseases as cancer. Deep learning methods occupy an increasing place in the processing of medical images [Qayyum]. They are used to improve the efficiency of segmentation, classification, and abnormality detection in images generated from different clinical imaging modalities including CT, MRI, X-rays, etc. In recent years, the advantages of deep learning have been experimentally revealed in comparison with other methods of machine learning in relation to medical image processing. In particular, it is shown that features created within deep learning methods significantly outperform the predictive ability of predefined features like tumor volume and maximum diameter [Hosny].

Progress in the development of deep learning methods is associated with convolutional neural networks $(\mathrm{CNN})$. In recent years, significant progress has been made in overcoming the main obstacle in applying CNN to medical images - the small amount of available medical data. UNet architecture [Ronneberger] has been developed, which allows network training on ultra-small datasets - up to several dozen of images. Generative adversarial networks (GAN), proposed in [Goodfellow], allow, in essence, to realize the synthesis of an unlimited number of images, which by statistical properties are arbitrarily close to the images from the training set. This property of GAN is already actively used in the formation of medical imaging datasets [Kazeminiaa], including for the diagnosis of lung cancer [Chuquicusma, Dakai].

Transfer learning [Weiss] also provides the ability to work with small datasets. This approach allows you to use $\mathrm{CNN}$, trained on a large source dataset, to work in another, target domain, by providing additional training on a small dataset containing data from the target domain. Transfer learning has proven to be an effective to working with medical images in CNN [Shin].

But lately, negative trends have been noted in the successful development of $\mathrm{CNN}$ in relation to medical images [Dong Wook Kim]. As practice shows, this development follows the path of increasing the complexity of the CNN architecture, which causes an extensive increase in the required computing resources 
(compare, for example, [Hammack] and [Karras]). There is a tendency to reduce the threshold for entering into the problems of medical image processing by creating more and more high-level and user-friendly software products and frameworks, but their full use requires more and more expensive hardware [Koryagin]. Finally, recent published studies are increasingly reporting on the extremely high efficiency of algorithms for the diagnostic analysis of medical images. However, according to leading journals [AI diagnostics], these results are often inspired by overfitting and are weakly related to the properties of datasets themselves, and therefore cannot be applied in clinical practice.

A promising way to overcome these trends is a more complete and effective account of the properties of the images themselves and the datasets collected from them, i.e. smarter data processing. Therefore, the attention of scientists is increasingly attracted by data processing methods, which make it possible to single out the design features characteristic of datasets themselves, and to rely on them when creating reliable and robust means of clinical diagnostics.

The article discusses two approaches to improving the efficiency of classification made by CNN associated with improved data processing, which we will consider on the example of an important problem - CT diagnosis of lung cancer. It is shown that even under resource constraints, they can give a gain in the efficiency of medical image analysis comparable to the use of "heavy" methods.

\section{DATA PRE-PROCESSING BASED ON ACTUAL DISTRIBUTION OF IMAGES' BRIGHTNESS}

\subsection{Background}

Preprocessing of medical images is meaningfully defined as a sequence of actions on newly received data that is executed on on newly received data in order to achieve the best fit with existing data [Lashari]. As a rule, the preprocessing of medical data used in CNN aims to accelerate convergence when training a network.

Various algorithms have been proposed for preprocessing medical images. The authors [Öztürka], aiming to regulate brightness and contrast variations in the image and suppresses noise, use different preprocessing algorithms and their combinations, including removing the median value or background from the original image as well applying median or wiener filter and adaptive threshold. In [Perumal], at the preprocessing stage of MRI images of lung cancer, the task of noise reduction is posed, and various filtering techniques like Wiener, Median, and Gaussian are used are used for this.

However, as shown in the literature [Vatian], noises in high-tech medical images, such as CT and MRI, have complex distribution patterns (a mixture of off-center gamma distributions for CT images and Rice distribution for MRI images), and attempts at noise filtration lead not only to the loss of semantically important details, but also to the appearance of artifacts.

Attempts are being made to take into account the complex nature of the brightness distributions of medical images. In [Zhenglun Kong] the authors constructed a histogram of MRI images of brain tissue and with the help of wavelet-transformation identified 4 distinct peaks in it. Using the obtained peak values as threshold values, they constructed a procedure for tissue clustering based on CNN. Such a preprocessing provided a proven increase in the reliability of the segmentation result. In [Joris] the authors investigated the histograms of CT and MRI brain images and established their heteroscedastic nature, that is varying degrees of statistical dispersion of tissue intensity distributions. In this regard, they propose to use non-linear transformation for image preprocessing, based on current estimate of of the intensity variance for each intensity value. The proposed algorithm improves the quality of segmentation of brain tissue measured by Dice scores.

However, despite their prospects, the proposed algorithms are quite sophisticated. In addition, we were unable to find similar approaches in the available literature in relation to CT images of the lungs. In general, as the reviews [Qayyum, Parmar] show, in the real practice of using CNN for processing medical images, the simplest methods prevail. These are data zero-centering and data normalization

$$
x^{\prime}=x-\bar{x}, \quad x^{\prime}=\frac{x-\bar{x}}{\sigma}, \quad \sigma=\frac{\sqrt{(x-\bar{x})^{2}}}{N-1},
$$


where $x$ is current pixel brightness, $\bar{x}$ and $\sigma$ are mean and stddev respectively, $\mathrm{N}$ is number of pixels in analyzed image(s). This approach obviously assumes that the original images obey a unimodal (usually Gaussian) distribution. However, if the assumption of unimodality is not justified in practice, it will not give the desired improvement in efficiency or may even worsen the situation. In connection with the above, in our work, we posed the following question: does the consideration of the specifics of the brightness distribution of CT images of lung cancer in its preprocessing affect the effectiveness of their classification by CNN?

\subsection{Materials and Method}

For research, we used data from dataset LUNA16 ${ }^{1}$. The original (total) dataset was divided into three parts, namely training, validation and test samples, with total amount of 6876, 1297 and 1622 images, 17.190.000, 3.242.500 and 4.055.000 pixels respectively. We built histograms of the brightness distributions of pictures and pixel brightness for each dataset separately and for all together, presented in figure 1, a-d. The variation of the pixel distributions of individual images is illustrated in Figure 2.

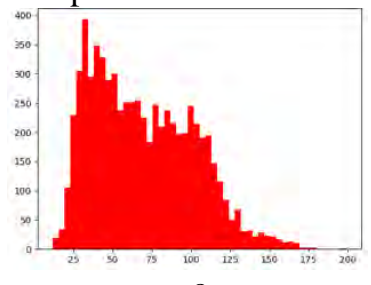

a

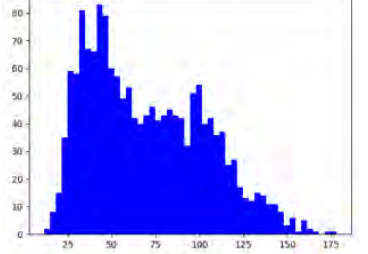

b

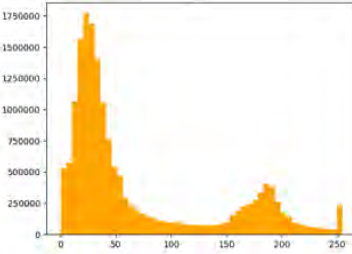

c

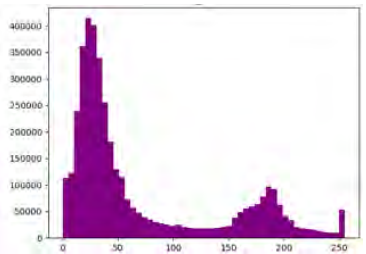

d

Figure 1. Images $(a, b)$ and pixel $(c, d)$ average brightness histograms for datasets $(a, c-$ train, b, d - test)
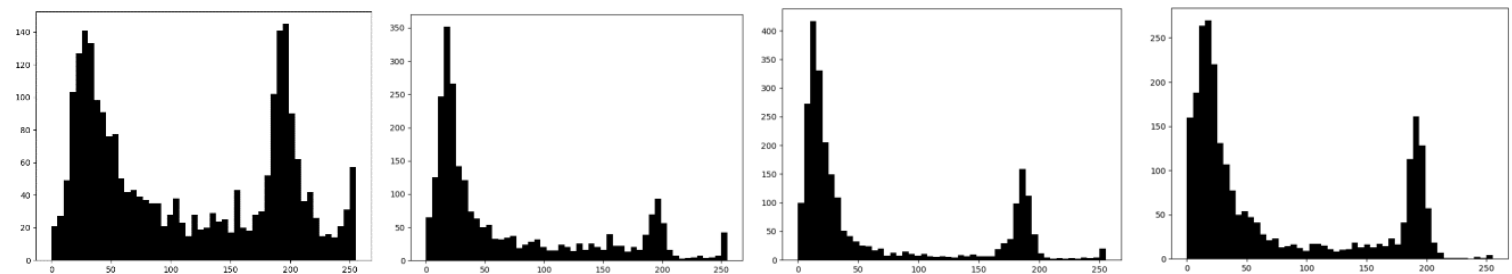

Figure 2. Examples of individual image brightness distributions

For a more subtle assessment of the brightness distributions, we divided the total pixel brightness range (0-255) into two subranges and built pixel brightness distributions for each subband. With a half division by brightness (into subbands $0-127$ and 128-255), we obtained almost identical results for all datasets, which are illustrated in Figure 3, a. When the boundary is shifted, the situation is qualitatively preserved, but the ratio of the maxima changes (see example in Figure 3, b). In Figure 3, c, it is possible to see a general estimate of the ratio of the magnitudes of the maxima of the distributions to the average brightness of the images. In our experiments, we used CNN with a rather simple architecture ${ }^{2}$ based on LeNet-5 architecture. Data preprocessing was performed in the following way. The array of pixels of each image was divided into subbands 0-127 (dark side) and 128-255 (light side). Mean and stddev values were calculated separately for the pixels of the dark side, the pixels of the light side, and for the entire image as a whole. Then we used three options:

Basic. The input of CNN is fed directly to the data from the total dataset.

Basic + mean/std. All pixels of each image are normalized to mean and stddev values according to (1) and (2).

Basic $+2 \mathrm{mean} / \mathrm{std}$. The pixels of the dark and light sides are separately normalized to the corresponding mean value and average deviation according to (1) and (2). Assuming that the brightness distributions of pixels on the dark and light sides are approximately normal, we used the " 3 sigma" rule for normalization, i.e. subtracted 3 from the dark part and added 3 to the light part.

\footnotetext{
${ }^{1}$ https://github.com/swethasubramanian/LungCancerDetection/tree/master/src/data

${ }^{2}$ http://datareview.info/article/kak-obuchit-glubokuyu-neyronnuyu-set-s-pomoshhyu-7-strok-koda/
} 

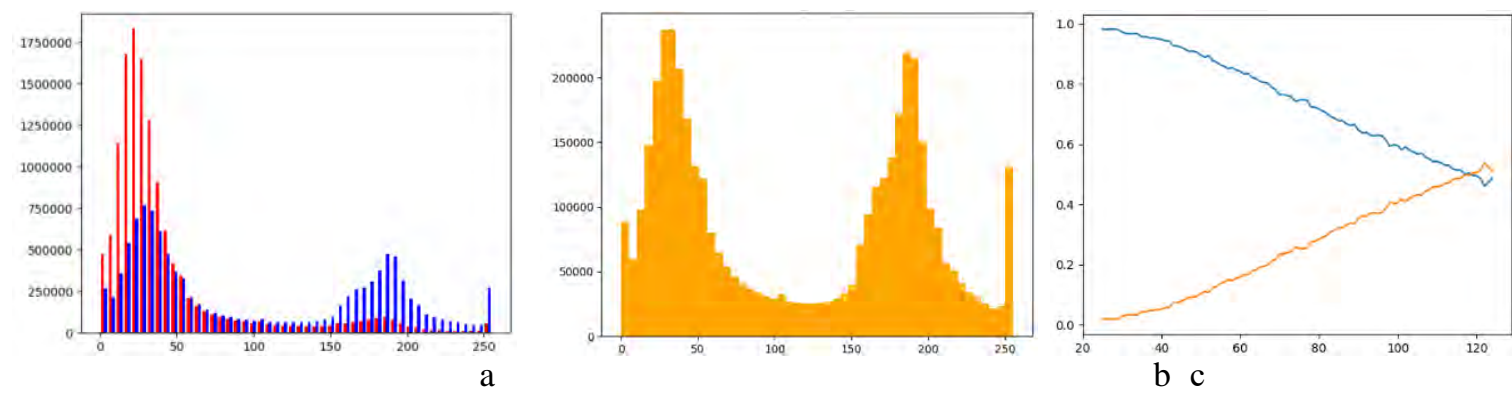

Figure 3. a - distributions of total dataset pixel brightness below average (red) and above average (blue); $b$ - distribution of train dataset pixels with brightness above the third quartile; $\mathrm{c}$ - dependence of the proportion of pixels with a value of $\geq 128$ (orange) and $<128$ (blue) on the average brightness of the picture

\subsection{Results and Discussion}

A comparative assessment of brightness distributions across all datasets constructed (Figure 1) shows that this distribution is rather far from normal. It is strongly scewed to the left, and there is some "dimple" near the mean value. The pixel brightness distribution (Figure 1) demonstrates an obvious bimodality with subsidence in the gray zone (127-128), and this distribution pattern is maintained for individual images (Figure 2). At the same time, the distribution components on the dark and light sides are quite close to normal (this assumption was successfully used in the organization of normalization in the Basic $+2 \mathrm{mean} / \mathrm{std}$ option).

Comparing histogrammes of individual images (Figure 2), we see that the absolute values of the maxima change, but their position remains unchanged. This regularity is confirmed on the average graphs (Figure 3, a, b). It is also worth noting the almost linear dependence of the number of "dark" (0-127) and "bright" (127-255) pixels on the average brightness of the image.

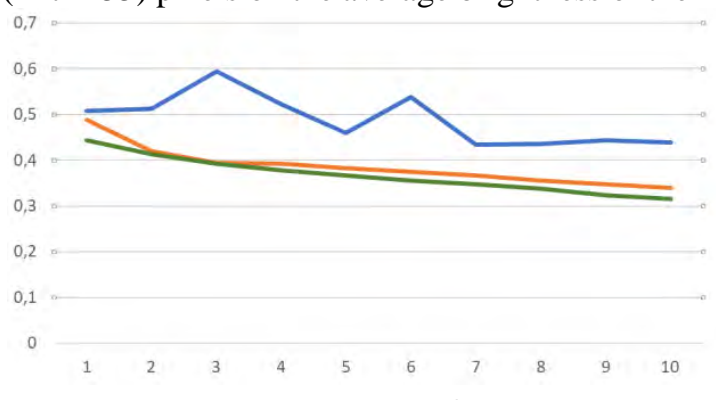

a

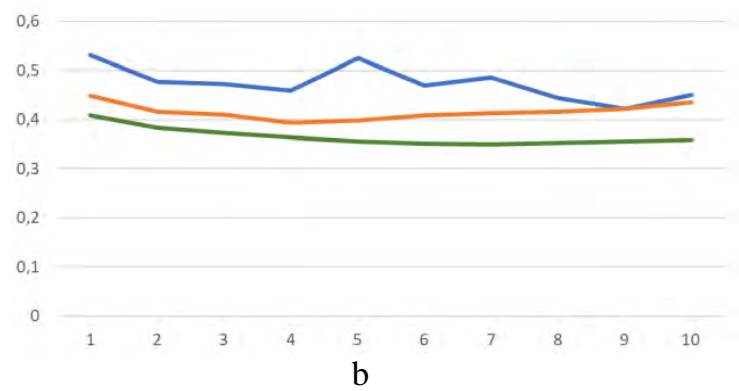

Figure 4. Dependence of loss function on the number of learning epochs for train (a) and test (b) datasets: blue - Basic option, orange - Basic + mean/std option, green - Basic +2 mean/std option
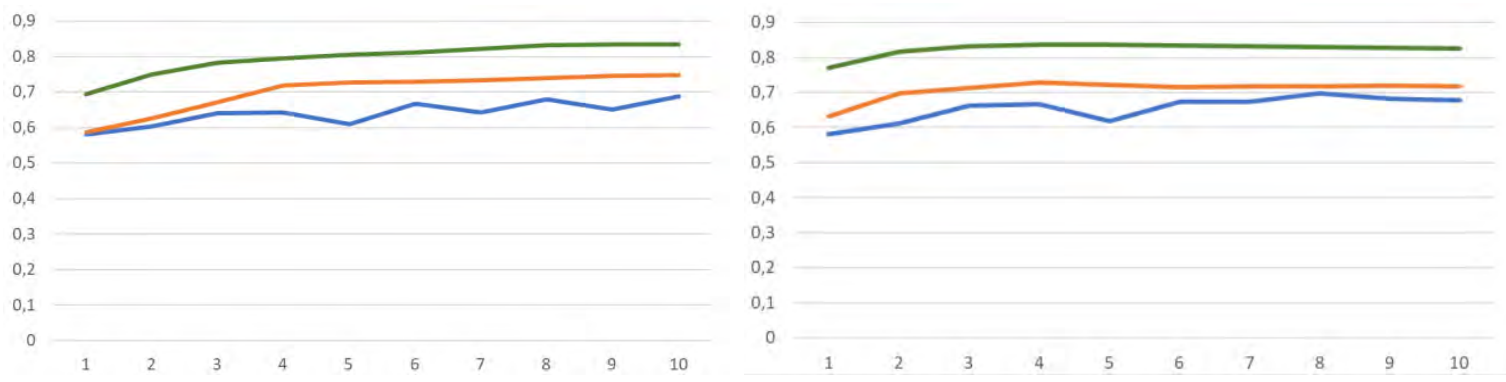

Figure 5. Dependence of roc_auc function on the number of learning epochs for train (a) and test (b) datasets: blue - Basic option, orange - Basic + mean/std option, green - Basic +2 mean/std option 
Graphs of the loss function and roc_auc on the training and validation samples, depending on the number of training epochs for each of the preprocessing options, are shown in Figure 4, a, b and Figure 5, a, b. Analysis of the graphs shows good convergence of results even with a small number of learning epochs, as well as the undoubted advantage of the Basic +2 mean / std option. We emphasize that these results were obtained with very scarce resource costs, both in terms of hardware and in terms of computing time, which allows us to give a confidently positive answer to the question posed in part 2.1.

\section{TRANSFER LEARNING BASED ON ACTUAL CORRELATION DEPENDENCIES IN IMAGES AND LATENT FEATURE SPACE}

\subsection{Background}

Transfer learning is one of the popular methods of using CNN to handle small datasets. Consider CNN solving the problem $\mathrm{T}=\{\mathrm{Y}, \ell(*)\}$ in the domain $\mathrm{D}=\{\mathrm{X}, \mathrm{P}(\mathrm{X})\}$. Let $\mathrm{D} \_$s be the source domain with the corresponding task T_s and D_t be the target domain (usually far smaller than D_s) with the task T_t. Then, according to [Weiss], transfer learning is the process of improving the function $\ell(*)$ for the $\mathrm{T}_{-} \mathrm{t}$ task using information from D_s. In order to do this, we first train CNN on a large dataset representing D_s. Then, leaving unchanged (freezing) the obtained weights of all layers of CNN, except the last, we replace the last layer with a new layer having the proper number of classes to solve the task T_t. Thereby we use the vector of high-level features formed by CNN on D_s to train last layer as a linear classifier for the problem T_t. In general, we can retrain not only the last layer, but also some of the previous ones, and with different learning rates. This technique is called fine tuning [Yosinski].

Transfer learning has already become the de facto standard in the processing of medical images for the diagnosis of lung cancer, as evidenced by the reviews and confirmed by experiments [Tao Tan, Nóbrega, Nishio]. However, the development of transfer learning in this area, as the analysis of the literature shows, goes mainly along the path of using more and more sofisticated CNNs and varying the parameters of layers being retrained. The statistical properties of the images themselves as a source of additional information about the relationship between D_s and D_t are practically not used. Meanwhile, within the framework of transfer learning, such opportunities exist, and one of them is provided by the DeepCoral algorithm.

DeepCORAL algorithm was proposed in [SunSaenko] as extension of the CORAL algorithm [SunFeng]. CORAL's key idea is to align second-order statistics of the source and target domain distributions. DeepCORAL extends this idea with the concept of training CNN's layers with an additional constraint on the alignment of the correlations of layer activations. For this for selected layers whose correlations are to be aligned, an additional loss function is introduced:

$$
\ell_{\text {CORAL }}=\frac{1}{4 d^{2}}\left\|C_{S}-C_{T}\right\|_{F}^{2},
$$

where \|\|$_{F}^{2}$ denotes the squared matrix Frobenius norm, and $C_{S}, C_{T}$ are the covariance matrices of the source and target data respectively. The CORAL losses collected from the selected layers are then summed and added to the classification loss:

$$
\ell=\ell_{\text {CLASS }}+\sum_{i=1}^{t} \lambda_{i} \ell_{\text {CORAL }}
$$

The gradient is then calculated for the backward propagation step for this ensembled loss function (4). As a result, CNN forms weights in feature space, the covariance of which does not correspond to the source domain D_s, but to the target domain D_t. These additional restrictions on the search conditions, as shown by experiments [SunSaenko], provide faster network convergence.

We were unable to find literature references about the use of the DeepCORAL algorithm in detecting lung cancer from CT images.

In connection with the above, in our work, we posed the following question: does the aligning of correlations of different datasets containing CT images of lung cancer affect the effectiveness of their classification by CNN? 


\subsection{Materials and Method}

As a source domain, we used the above mentioned dataset LUNA16. As a referent target domain, we used lung nodule dataset of $\mathrm{SPIE}^{3}$. As a target domain of interest, we used a new dataset, referred as Medical, formed by clinicists with strict control of CT parameters during lung cancer treatments (see Table 1 for details). As can be seen from the table 1, Medical dataset is much more statistically homogeneous than LUNA, which allows to consider them as different domains in our experiments.

Table 1. Comparison of datasets parameters

\begin{tabular}{lccccc}
\hline Dataset & $\begin{array}{c}\text { Scans / patients in } \\
\text { total }\end{array}$ & $\begin{array}{c}\text { Types of } \\
\text { scanners }\end{array}$ & $\begin{array}{c}\text { Variants of tube peak } \\
\text { potential }\end{array}$ & $\begin{array}{c}\text { Variants of slice } \\
\text { thicknesses }\end{array}$ & $\begin{array}{c}\text { Reconstruction } \\
\text { interval }\end{array}$ \\
\hline LUNA16 & $1018 / 1010$ & 6 & 4 & 11 & $0.45-5.0 \mathrm{~mm}$ \\
MEDICAL & $20 / 20$ & 1 & 1 & 1 & $1.25 \mathrm{~mm}$ \\
\hline
\end{tabular}

As a reference method of transfer learning we used fine tuning. We used a lung cancer-specific architecture $^{4}$ that is a modification of the UNet architecture for segmentation of 3D objects [Fangzhou Liao]. We implement the fine tuning method in a traditional way:

- Remove the last fully connected layers of the original NA

- Adding own fully connected layers with number of classes according to $\mathrm{T}$ t , corresponding to benign and malignant nodules.

- Freezing of convolutional layers of the initial network.

- Training added layers

- Unfreeze the rest layers of the original network.

- Further training of the entire network with added layers.

To implement the DeepCORAL algorithm, we used AlexNet architecture as a base with the replacement of the activation functions ReLU with the ELU (Clevert, 2015) and the addition of BatchNorm layers (Ioffe \& Szegedy, 2015), which is more relevant in modern industry practice. We conducted experiments to optimize the number of layers to be aligned and found that the best result, in contrast to the original architecture [27], gives the alignment of all five convolutional layers in AlexNet and two of the three dense layers, except the last.

\subsection{Results and Discussion}

The limiting values of accuracy obtained by us using the reference method of fine tuning in the retraining from LUNA16 to SPIE and MEDICAL, respectively, are presented in Table 2.

Table 2. Accuracy of lung cancer detection using the reference method of fine tuning

\begin{tabular}{lcc}
\hline Dataset & w/o Fine Tuning, $\%$ & with Fine Tuning, $\%$ \\
\hline SPIE & 63 & 85 \\
MEDICAL & 61 & 73 \\
\hline
\end{tabular}

We see an improvement in the accuracy up to values comparable to the level of recent achievements [Nishio], however, this improvement was achieved due to the large resource costs: the additional training of our reference network to obtain mentioned results took about two weeks on two GTX 1070 GPUs.

Figure 6 shows the results of retraining from source dataset LUNA16 to target dataset MEDICAL using DeepCORAL algorithm. Comparing figure 6, c, and figure 6, f, we see that expressive power of used AlexNet is not enough to make correct predictions on the target dataset, and ROC-AUC score oscillates around 0.5 , nearly at random prediction level. In the same time, using the same CNN with the integrated DeepCORAL gives more accurate predictions for the target dataset, and even at $70^{\text {th }}$ training epoch trend of ROC-AUC score still remains positive. Note that each experiment with network training for 70 epochs took only about an hour on the one GTX 1070 GPU.

\footnotetext{
${ }^{3}$ https://www.spiedigitallibrary.org/conference-proceedings-of-spie/7260/1/The-Lung-TIME--annotated-lung-nodule-dataset-andnodule/10.1117/12.811645.short?SSO=1

${ }^{4}$ https://www.kaggle.com/c/data-science-bowl-2017
} 

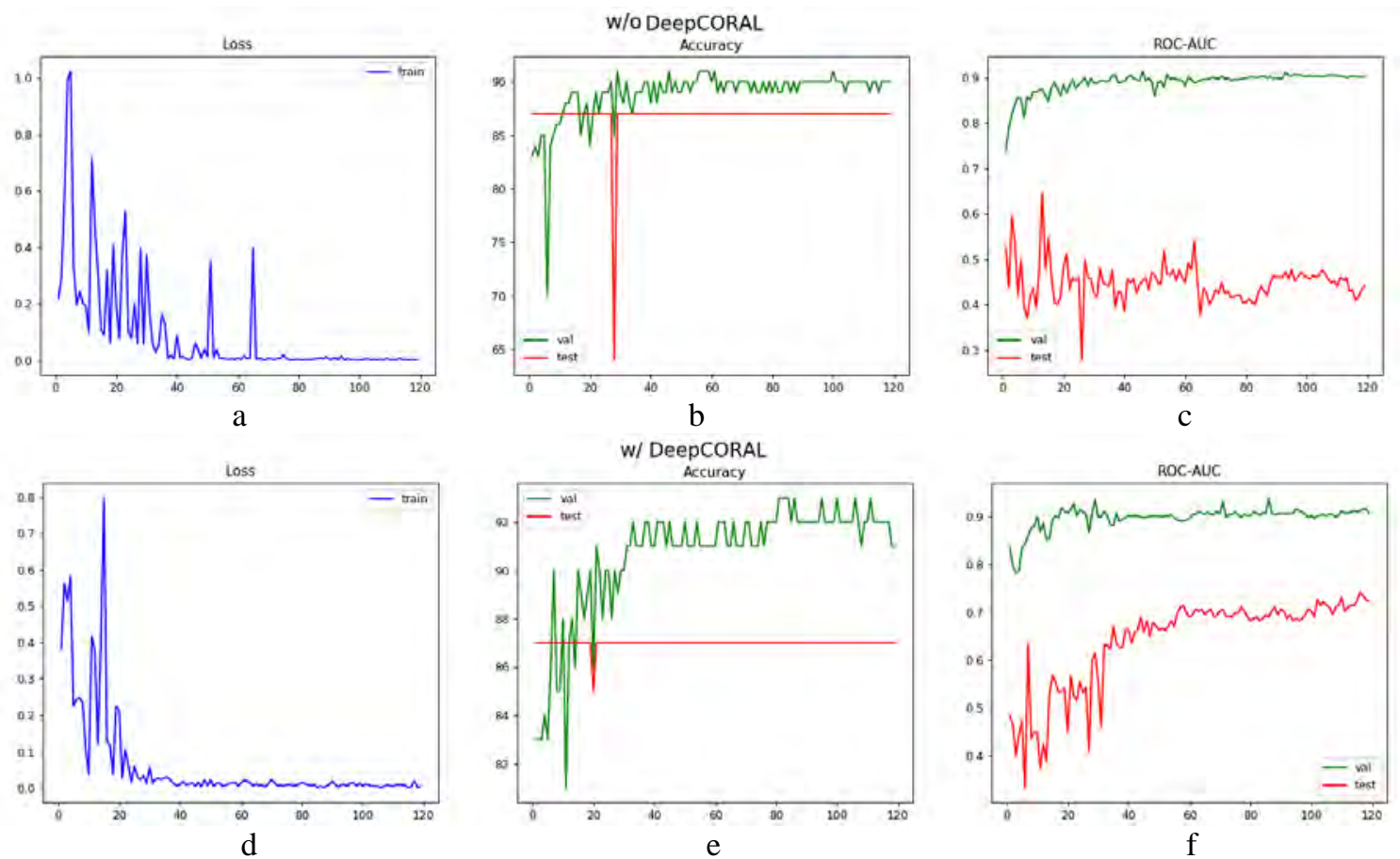

Figure 6. Dependencies of loss, accuracy and ROC-AUC on the number of training epochs not using (a-c) and using $(d-f)$ DeepCORAL. The green line is scores for the validation subset of the source dataset, and the red line is the same for the target dataset

Thus, we have shown that using the DeepCoral method which takes into account the correlation between the images of the source and target domains for transfer learning allows us to obtain a quality of classification comparable to results of fine-tuning on a much more powerful CNN and at 120 epochs, but with a strong decrease in the required computational and time resources. It allows to give a confidently positive answer to the question posed in part 3.1.

\section{CONCLUSION}

The paper proposes two approaches to improving the efficiency of the classification of CT images of lung tumors, performed using CNN. These approaches are associated with improving of data processing and network training method. Data processing approach takes into account the current brightness distributions of CT scans, which showes better training results in the experiments. The network training method considers the integration of CORAL loss into network architecture, which provides the aligning of latent feature space of the CNN trained on source large dataset of CT scans with the latent feature space of the target small dataset, where the network will be used for inference. This method shows results comparable with the traditional fine-tuning method on the same hardware resources but on in a much shorter training time. In future work, we are going to use these approaches in more complex $\mathrm{CNN}$ architectures and we expect that this ratio should survive.

This work was financially supported by the Government of Russian Federation, Grant 08-08.

\section{REFERENCES}

AI diagnostics need attention. Nature 2018;555:285.

Chuquicusma M.J.M., Hussein S., Burt J., Bagci U. How to Fool Radiologists with Generative Adversarial Networks? A Visual Turing Test for Lung Cancer Diagnosis. posarXiv: 1710.09762v2 [cs.CV] 9 Jan 2018

Dakai Jin, Ziyue Xu, Youbao Tang, Harrison A.P., Mollura D.J. CT-Realistic Lung Nodule Simulation from 3D Conditional Generative Adversarial Networks for Robust Lung Segmentation arXiv:1806.04051v1 [cs.CV] 11 Jun 2018 
Dong Wook Kim, Hye Young Jang, Kyung Won Kim, Youngbin Shin, Seong Ho Park. Design Characteristics of Studies Reporting the Performance of Artificial Intelligence Algorithms for Diagnostic Analysis of Medical Images: Results from Recently Published Papers // Korean J Radiol. 2019 Mar; 20(3)/ Pp. 405-410.

Fangzhou Liao, Ming Liang, Zhe Li, Xiaolin Hu, Sen Song. Evaluate the Malignancy of Pulmonary Nodules Using the 3D Deep Leaky Noisy-or Network // Journal Of Latex Class Files, Vol. 14, No. 8, August 2015.

Goodfellow I., Pouget-Abadie J., Mirza M., Xu B., Warde-Farley D., Ozair S., Courville A., Bengio Y. Generative adversarial nets // Advances in neural information processing systems, 2014, pp. 2672-2680.

Hammack D. Forecasting Lung Cancer Diagnoses with Deep Learning // Data Science Bowl 2017 Technical Report, April 22, 2017

Hosny A., Parmar C., Coroller T.P., Grossmann P., Zeleznik R., Kumar A., Bussink J., Gillies R.J., Mak R.H., Aerts J.W.L. Deep learning for lung cancer prognostication: A retrospective multi-cohort radiomics study // PLoS Med. 2018 Nov; 15(11): e1002711. Published online 2018 Nov 30. doi: 10.1371/journal.pmed.1002711

Joris P., Develter W., Van De Voorde W., Suetens P., Maes F., Vandermeulen D., Claes P. Preprocessing of Heteroscedastic Medical Images // IEEE Access, V. 6, 2018. Pp. 26047-26058.

Karras T., Aila T., Laine S., Lehtinen J. Progressive Growing Of Gans For Improved Quality, Stability, And Variation // ICLR 2018 Conference, Feb 2018.

Kazeminiaa S., Baurb C., Kuijperc A., van Ginnekend B., Navabb N., Albarqounib S., Mukhopadhyaya A. GANs for Medical Image Analysis. 21 Dec 2018. https://arxiv.org/abs/1809.06222

Koryagin A. Automatic lung-cancer detection on scans of Computed Tomography with RadIO. Feb. 2018. Availible: https://medium.com/data-analysis-center/automatic-lung-cancer-detecion-on-scans-of-computed-tomography-withradio-945d781aa022

Lashari S.A., Ibrahim R. A Framework for Medical Images Classification Using Soft Set. // Procedia Technology $11(2013) 548-556$

Nishio M., Sugiyama O., Yakami M., Ueno S., Kubo T., Kuroda T., et al. (2018) Computer-aided diagnosis of lung nodule classification between benign nodule, primary lung cancer, and metastatic lung cancer at different image size using deep convolutional neural network with transfer learning. // PLoS ONE 13(7): e0200721. https://doi.org/10.1371/journal.pone.0200721

Nóbrega R. V., Peixoto S. A., da Silva S. P. P., Rebouças Filho P. P. Lung Nodule Classification via Deep Transfer Learning in CT Lung Images // 2018 IEEE 31st International Symposium on Computer-Based Medical Systems (CBMS). 18-21 June 2018

Öztürka Ş., Akdemir B. Effects of Histopathological Image Pre-processing on Convolutional Neural Networks // Procedia Computer Science 132 (2018) 396-403

Parmar C., Barry J.D., Hosny A., Quackenbush J., Aerts H.J.W.L. Data Analysis Strategies in Medical Imaging // Clin Cancer Res; 24(15) August 1, 2018

Perumal S. and Velmurugan T. Preprocessing by Contrast Enhancement Techniques for Medical Images // International Journal of Pure and Applied Mathematics. Volume 118. No. 18, 2018, 3681-3688

Qayyum A., Anwar S.M., Majid M., Awaisc M., Alnowamid M. Medical Image Analysis using Convolutional Neural Networks: A Review // Journal of Medical Systems. October 2018. DOI: 10.1007/s10916-018-1088-1

Ronneberger O., Fischer P., Brox T. U-Net: Convolutional Networks for Biomedical Image Segmentation. May 2015. https://arxiv.org/abs/1505.04597

Shin Hoo-Chang et al. Deep Convolutional Neural Networks for Computer-Aided Detection: CNN Architectures, Dataset Characteristics and Transfer Learning // IEEE Trans Med Imaging. 2016 May; 35(5): 1285-1298. doi:10.1109/TMI.2016.2528162.

Sun B., Feng J., Saenko, K. Return of frustratingly easy domain adaptation // AAAI. (2016)

Sun B., Saenko K. Deep CORAL: Correlation Alignment for Deep Domain Adaptation // arXiv:1607.01719v1 [cs.CV] 6 Jul 2016

Tao Tan, Zhang Li, Haixia Liu et al. Optimize transfer learning for lung diseases in bronchoscopy using a new concept: sequential fine-tuning // arXiv:1802.03617v1 [cs.CV] 10 Feb 2018

Vatian A., Gusarova N., Dobrenko N., Dudorov S., Nigmatullin N., Shalyto A., Lobantsev A. Impact of Adversarial Examples on the Efficiency of Interpretation and Use of Information from High-Tech Medical Images // FRUCT 2019

Weiss K., Khoshgoftaar T., DingDing Wang. A survey of transfer learning // Journal of Big Data (2016) 3:9 DOI 10.1186/s40537-016-0043-6

Yosinski J., Clune J., Bengio Y., Lipson H. How transferable are features in deep neural networks? // Advances in Neural Information Processing Systems 27 (NIPS '14), NIPS Foundation, 2014.

Zhenglun Kong, Ting Li, Junyi Luo, Shengpu Xu. Automatic Tissue Image Segmentation Based on Image Processing and Deep Learning // Journal of Healthcare Engineering. Volume 2019, Article ID 2912458, 10 pages 